\title{
Quasiquadrature Solution of the Linear Integral Equations of the Second Kind in the Class of Integrable Functions
}

\author{
Igor Petrovich Dobrovolsky \\ Institute of Physics of the Earth, RAS, Moscow, Russia \\ Email:dipedip@gmail.com
}

How to cite this paper: Dobrovolsky, I.P (2020) Quasiquadrature Solution of the Linear Integral Equations of the Second Kind in the Class of Integrable Functions. Open Access Library Journal, 7: e6069. https://doi.org/10.4236/oalib.1106069

Received: January 13, 2020

Accepted: February 10, 2020

Published: February 13, 2020

Copyright $\odot 2020$ by author(s) and Open Access Library Inc.

This work is licensed under the Creative Commons Attribution International License (CC BY 4.0).

http://creativecommons.org/licenses/by/4.0/

\begin{abstract}
The analog of the quadrature solution of the equation of the second kind is considered. Fundamental difference from classical quadrature formulas is as follows. On segments of the chosen grid not values of functions, but their integral average values are used. Equations of Fredholm and Volterra are considered. The graphical representation of the solution is discussed. Computing examples show expediency of such approach in appropriate cases.
\end{abstract}

\section{Subject Areas}

Integral Equation

Keywords

Integral Average Value

\section{Introduction}

Numerical methods of the solution of the equations are universal. This is their important quality. Quadrature formulas are quite often used in the solution of the integral equations. Quadrature formulas are applied only to continuous functions. In other words, the integrable function has to have a certain value at each point. This requirement cannot be fulfilled for the class of integrable functions. In such situation the alternative version of the quadrature solution of the integral equation is possible. This work is further work [1].

For convenience of readers in this work, fundamentals of the article [1] are repeated. 


\section{Fredholm's Equation}

The essence of the offered algorithm is as follows. On the main segment of the integral equation the system of segments is formed and on each such segment the integral average value of function is defined.

The equation is considered

$$
y(x)-\int_{a}^{b} K(x, t) y(t) \mathrm{d} t=f(x)
$$

which has the unique solution in a class of the integrable functions.

We form on the segment $[a, b]$ a uniform grid of $N$ segments with a step

$$
h=\frac{b-a}{N}
$$

On the grid there is $N+1$ of points. We build segments $\delta_{i}$ in the vicinity of each point

$$
\begin{aligned}
& \delta_{0}=\left[a, a+\frac{h}{2}\right], \delta_{i}=\left[a+\frac{h}{2}(2 i-1), a+\frac{h}{2}(2 i+1)\right], i=1, \cdots, N-1, \\
& \delta_{N}=\left[b-\frac{h}{2}, b\right]
\end{aligned}
$$

We will enter concept of integral average values on these segments. They have the form or functions $y(x)$ and $f(x)$

$$
\begin{aligned}
& w_{i}=\frac{1}{\delta_{i}} \int_{\delta_{i}} y(x) \mathrm{d} x, \\
& f_{j}=\frac{1}{\delta_{j}} \int_{\delta_{j}} f(x) \mathrm{d} x
\end{aligned}
$$

Let's provide the function $y(t)$ under integral (2.1) in the form

$$
y t=\sum_{i=0}^{N} w_{i} \delta_{i}
$$

where $\delta_{i}$ is characteristic function of the corresponding segment.

Then (2.1) receives the form

$$
y(x)-\sum_{i=0}^{N} K_{i}(x) w_{i}=f(x)
$$

where $K_{i}(x)=\int_{\delta_{i}} K(x, t) \mathrm{d} t$.

Let's carry out to (2.6) integral averaging on segments $\delta_{j}$

$$
w_{j}-\sum_{i=1}^{N} K_{j i} w_{i}=f_{j}, j=0, \cdots, N
$$

where $K_{j i}=\frac{1}{\delta_{j}} \int_{\delta_{j}} \int_{\delta_{i}} K(x, t) \mathrm{d} t \mathrm{~d} x, f_{j}=\frac{1}{\delta_{j}} \int_{\delta_{j}} f(x) \mathrm{d} x, j$ is number of the equation, $i$ is number of item in the equation.

(2.7) is system of the equations for definition of integral average values of unknown function on system of segments (2.3). 


\section{Volterra's Equation}

The equation is considered

$$
y(x)-\int_{0}^{x} K(x, t) y(t) \mathrm{d} t=f(x)
$$

For the given $h$ we accept the grid of segments

$$
\delta_{1}=\left[0, \frac{h}{2}\right], \delta_{i}=\left[\frac{h}{2}(2 i-3), \frac{h}{2}(2 i-1)\right], i=2,3, \cdots
$$

Let's consider two grids (3.2) with steps of $h$ and $h / 2$. Then the system of the bisecting points of the whole segments of the first grid completely contains in a system of the bisecting points of the second grid. Thanks to it, it is possible to carry out their comparison and processing.

We accept $y=w_{1} \delta_{1}$ on the first step under integral in (3.1). After averaging on $x$ of the received equation on the segment $\delta_{1}$ it is possible to calculate $w_{1}$

$$
w_{1}=\frac{f_{1}}{1-K_{1}}
$$

where $f_{1}=\frac{2}{h} \int_{0}^{h / 2} f(x) \mathrm{d} x$ and $K_{1}=\frac{2}{h} \int_{0}^{h / 2} \int_{0}^{x} K(x, t) \mathrm{d} t \mathrm{~d} x$.

For further calculations we introduce designations

$$
\begin{aligned}
& f_{n}=\frac{1}{h} \int_{h(2 n-3)}^{h(2 n-1) / 2} f(x) \mathrm{d} x, n=2,3, \cdots \\
& k_{n, 1}=\frac{1}{h} \int_{h(2 n-3) / 2}^{h(2 n-1) / 2} \int_{0}^{h / 2} K(x, t) \mathrm{d} t \mathrm{~d} x, n=2,3, \cdots \\
& K_{n, i}=\frac{1}{h} \int_{h(2 n-3) / 2}^{h(2 n-1) / 2} \int_{h(2 i-3) / 2}^{h(2 i-1) / 2} K(x, t) \mathrm{d} t \mathrm{~d} x, \quad n=3,4, \cdots, n>i \\
& k_{n, n}=\frac{1}{h} \int_{h(2 n-3) / 2}^{h(2 n-1) / 2} \int_{h(2 n-3) / 2}^{x} K(x, t) \mathrm{d} t \mathrm{~d} x, n=2,3, \cdots
\end{aligned}
$$

On the second step the Equation (3.1) receives the form

$$
w_{2}-w_{1} k_{2,1}-w_{2} k_{2,2}=f_{2}
$$

from where

$$
w_{2}=\frac{f_{2}+w_{1} k_{2,1}}{1-k_{2,2}}
$$

On the third and the subsequent steps we receive

$$
w_{n}=\frac{f_{n}+w_{1} k_{n, 1}+\sum_{i=2}^{n-1} w_{i} K_{n, i}}{1-k_{n, n}}
$$

The linear change of variables transforms integrals with variable limits into integrals with constant limits

$$
\int_{c}^{d} \int_{a(x)}^{b(x)} f(x, t) \mathrm{d} t \mathrm{~d} x=\int_{c}^{d} \int_{0}^{1}(b-a) f(x, y(b-a)+a) \mathrm{d} y \mathrm{~d} x
$$


which are calculated by means of the known algorithms.

\section{Graphical Representation and Possibility of Refinement of the Solution}

In the quasiquadrature solution integral average value cannot be referred to any certain point of the segment and it is possible to accept that function is constant on each segment. Therefore, the graph of the quasiquadrature solution is step function, i.e. the histogram. It is reasonable to consider that integral average value is value in the central point of each segment and to build the graph using this assumption.

On segments where function is rather smooth, it is possible to construct algorithm for increase in accuracy of solutions. Let's consider such segment. We will enter on this segment the new coordinate $z=x-x_{0}$ where $x_{0}$ is bisecting point of a segment. Let's construct system of segments $h_{i}$ with the general center $z=0$. We assume $h_{i+1}<h_{i}$ and $h_{i} \rightarrow 0$ at $i \rightarrow \infty$. Obviously

$$
\frac{1}{h_{i}} \int_{-h_{i} / 2}^{h_{i} / 2} y(z) \mathrm{d} z \rightarrow y(0)=w \text { at } i \rightarrow \infty
$$

where $w$ is exact value of the integral average value in the vicinity of the point of $z=0$.

According to Weierstrass's theorem continuous function can be approximated arbitrary exactly by polynom and we will present on the segment $h_{1}$ the function $y(z)$ in the form

$$
y(z)=y(0)+\sum_{k=1} a_{k} z^{k}
$$

where lack of the top sign at the sum means that the remainder term is included in this sum.

Then

$$
\frac{1}{h_{i}} \int_{-h_{i} / 2}^{h_{i} / 2} y(z) \mathrm{d} z=y(0)+\sum_{k=1} \frac{a_{2 k}}{4^{k}(2 k+1)} h_{i}^{2 k}=y(0)+\sum_{k=1} b_{k} h_{i}^{2 k}=w_{i}
$$

Now (4.3) taking into account (4.1) and (4.2) it is possible to write down in the form

$$
w-w_{i}=\sum_{k=1} b_{k} h_{i}^{2 k}
$$

Expression (4.4) matches the formula (2.1) in [2] which is used for carrying out extrapolation of Richardson, and it is possible to use the results received in [2]. In [2] value $w_{i}=w_{i}^{(0)}$ is called extrapolation of the zero order. For the most important case when

$$
h_{i+1}=\frac{h_{i}}{2}
$$

we have the recurrence formula

$$
w_{i}^{(j+1)}=\frac{4^{j+1} w_{i+1}^{(j)}-w_{i}^{(j)}}{4^{j+1}-1}
$$


Richardson's extrapolation is effective only at sufficient smoothness of the sought function. However, there is no need to conduct special research of smoothness of function: from the extrapolation table and derivative tables [2] will automatically be defined to what order of extrapolation is effective.

\section{Example. Fredholm's Equation}

The equation is considered

$$
y(x)-\int_{0}^{1} \sqrt{|t-x|} y(t) \mathrm{d} t=\frac{1}{\sqrt{x}}-\frac{x \pi}{2}-\frac{x \ln x}{2}-\sqrt{1-x}+\frac{1}{2} x \ln (2-x+2 \sqrt{1-x})
$$

which has the exact solution $y=\frac{1}{\sqrt{x}}$.

Results of the quasiquadrature solution of this equation are presented in Table 1.

In Table $1 y_{0}$ and $y_{N}$ are the integral average values calculated according to the exact solution.

Let's make some analysis of the received solution. We see that the function $y(x)$ grows at approach to a point $x=0$. We will assume that this growth has power character. Specifically: we will assume that in the vicinity of a point $x=0$ function has form $p+q x^{n}$. Then

$$
\frac{2}{h} \int_{0}^{h / 2}\left(p+q x^{n}\right) \mathrm{d} x=p+\frac{q h^{n}}{(n+1) 2^{n}}=p+\frac{q}{2^{n}(n+1) N^{n}}
$$

For definition of unknown $p q$ and $n$ we equate the received expression to three values from Table 1

$$
\begin{aligned}
& p+\frac{q}{2^{n}(n+1) 160^{n}}=w_{0}(160)=35.777063 \\
& p+\frac{q}{2^{n}(n+1) 240^{n}}=w_{0}(240)=43.817803 \\
& p+\frac{q}{2^{n}(n+1) 320^{n}}=w_{0}(320)=50.596448
\end{aligned}
$$

The solution of system (5.3) gives (after rounding) $n=-0.5, p=0, q=1$. This solution correctly defines behavior of function $y(x)$.

Table 1. Quasiquadrature solution of Equation (5.1).

\begin{tabular}{ccccc}
\hline \multirow{2}{*}{$N$} & \multicolumn{2}{c}{ segment $\delta_{0}$} & \multicolumn{2}{c}{ segment $\delta_{N}$} \\
\cline { 2 - 5 } & $w_{0}$ & $y_{0}$ & $W_{N}$ & $y_{N}$ \\
\hline 20 & 12.647499 & 12.649110 & 1.00249 & 1.00632 \\
40 & 17.888020 & 17.888543 & 1.00166 & 1.00314 \\
80 & 25.298077 & 25.298221 & 1.00100 & 1.00156 \\
160 & 35.777063 & 35.777088 & 1.00057 & 1.00078 \\
240 & 43.817802 & 43.817805 & 1.00041 & 1.00052 \\
320 & 50.596448 & 50.596443 & 1.00031 & 1.00039 \\
\hline
\end{tabular}


Table 2. Quasiquadrature solution of Equation (6.1).

\begin{tabular}{ccccc}
\hline \multirow{2}{*}{$h$} & \multicolumn{2}{c}{ the first segment } & \multicolumn{2}{c}{ segment at $x=1$} \\
\cline { 2 - 5 } & $W_{1}$ & $y_{1}$ & $W_{N}$ & $y_{N}$ \\
\hline $1 / 50$ & 0.067869079 & 0.066666667 & 1.006032 & 0.999996 \\
$1 / 100$ & 0.047711701 & 0.047140452 & 1.001618 & 0.999999 \\
$1 / 200$ & 0.033610541 & 0.033333333 & 1.000424 & 1 \\
$1 / 400$ & 0.023705989 & 0.023570226 & 1.000110 & 1 \\
$1 / 600$ & 0.019334704 & 0.019245009 & 1.000050 & 1 \\
$1 / 800$ & 0.016733578 & 0.016666667 & 1.000028 & 1 \\
\hline
\end{tabular}

\section{Example. Volterra's Equation}

The equation is considered

$$
y(x)-\int_{0}^{x} \frac{y(t) \mathrm{d} t}{\sqrt{x-t}}=\sqrt{x}-\frac{x \pi}{2}
$$

which has the exact solution $y=\sqrt{x}$.

Results of the quasiquadrature solution of this equation are presented in Table 2.

In Table $2 y_{0}$ and $y_{N}$ are the integral average values calculated according to the exact solution. Using a formula (5.2) it is also possible to carry out the analysis at vicinity of a point of $x=0$.

\section{Conclusion}

The presented work and work [2] liquidate the last gaps in the field of quadrature solutions of linear integral equations of the second kind.

\section{Conflicts of Interest}

The author declares no conflicts of interest regarding the publication of this paper.

\section{References}

[1] Dobrovolsky, I.P. (2019) Quasi-Quadrature Solution of Integral Equations Fredholm of the Second Kind in the Class of Integrable Functions. Open Access Library Journal, 6, e5736. https://doi.org/10.4236/oalib.1105736

[2] Dobrovolsky, I.P. (2015) The Estimation of the Error at Richardson's Extrapolation and the Numerical Solution of Integral Equations of the Second Kind. Open Access Library Journal, 2, e2051. https://doi.org/10.4236/oalib.1102051 